\title{
The Necessity of Standards in an ISO 17025 Accredited Bioforensic Electron Microscopy Laboratory
}

Brian M. Leroux ${ }^{1}$ and Robert K. Pope ${ }^{1}$

${ }^{1}$ National Bioforensic Analysis Center, 8300 Research Plaza, Fort Detrick, MD, USA.

The use of standards and the development of validated methods of sample processing and analysis for microscopy increases the repeatability, reliability, consistency, and overall quality of output. ISO 17025 accreditation ensures that all personnel are competent to perform assays, all assays are validated to perform properly in the specific space accredited, and that the laboratory as a whole is proficient in the performance of the assay. Additionally, ISO 17025 accreditation ensures that critical supplies are from approved vendors and equipment is maintained properly. This includes the use of traceable ASTM standards, quality controls and a quality management system [1]. ISO 9001 is a set of standards and guidelines for good quality management practices, while ISO 17025 goes beyond the ISO 9001 standard to include additional mechanisms for quality control, document control, quality analysis, and trending of data. The National Bioforensic Analysis Center's bioforensic electron microscopy laboratory is ISO 17025 accredited to analyze samples using Scanning Electron Microscopy (SEM), Transmission Electron Microscopy (TEM), and Energy Dispersive X-ray Spectroscopy (EDS). When used properly, the inclusion of standards and approved methods provide additional layers of quality to increase the validity of the data in law enforcement court proceedings.

TEM verification is conducted with National Institute of Standards and Technology (NIST) traceable Nanosphere standards (Figure 1A). These are verified upon arrival with a lot test, and subsequently analyzed prior to the imaging of casework samples. Images of the spheres are analyzed and measured in an $\mathrm{X}$ and $\mathrm{Y}$ orientation, and the scale bar and magnification are subsequently verified to be correct. All of this is documented on the TEM per use performance check form. While there are other TEM standards available, these are NIST traceable and meet the ISO 17025 standard for magnification verification.

Standards for SEM analysis must demonstrate that the microscope is functioning properly and that the software used for capturing images is producing the correct magnification. The largest hurdle was finding a standard that could be ISO 17025 verified. For our purposes, we use the Geller MRS-5 standard (Figure 1B) that can be sent for recalibration. Before the analysis of casework samples, images of the Geller standard are captured. These images must demonstrate correct measurements on both the $\mathrm{X}$ and $\mathrm{Y}$ axis, and correct measurement of the scale bar and a correct magnification must be produced by the software. This demonstrates that the microscope is functioning properly, that the scale bar and magnification produced by the software are correct, and is documented on the SEM by use of a performance check form.

For EDS, our assay was based on the ASTM E-1508-12a procedure [2]. Two verifications must occur prior to the analysis of casework samples. The first is the verification that the detector is capturing the data correctly (documented with a K line for copper at 8.04 +/- 0.1, Fig 1C) using the NIST SRM 482 Pure Element Au-Cu Alloy System. The second is that the software is interpreting the data correctly. This is documented with the concentration of manganese (should be at $1.64 \%+/-4 \%(1.57 \%$ to $1.71 \%$, Fig 1D) in the Stainless Steel SRM 1155 on the Pelco ${ }^{\circledR}$ XCS-5 standard.

The use of reference materials, verified procedures, controlled documents, traceable reagents, traceable equipment maintenance records, and competency tested laboratory personnel all help to support the 
validity of any data produced. This also reduces the potential for using non-approved supplies, equipment and personnel, ensuring the data produced is of the highest quality [3].

References:

[1] ANSI/ISO/IEC 17025: 2005 “General Requirements for the Competence of Testing and Calibration Laboratories."

[2] ASTM E1508-12a (2012) Standard Guide for Quantitative Analysis by Energy-Dispersive Spectroscopy, ASTM International, PA

[3] This work was funded under Contract No. HSHQDC-15-C-00064 awarded by the Department of Homeland Security (DHS) Science and Technology Directorate (S\&T) for the operation and management of the National Biodefense Analysis and Countermeasures Center (NBACC), a Federally Funded Research and Development Center. The views and conclusions contained in this document are those of the authors and should not be interpreted as necessarily representing the official policies, either expressed or implied, of the DHS or S\&T. In no event shall DHS, NBACC, S\&T or Battelle National Biodefense Institute have any responsibility or liability for any use, misuse, inability to use, or reliance upon the information contained herein. DHS does not endorse any products or commercial services mentioned in this publication.

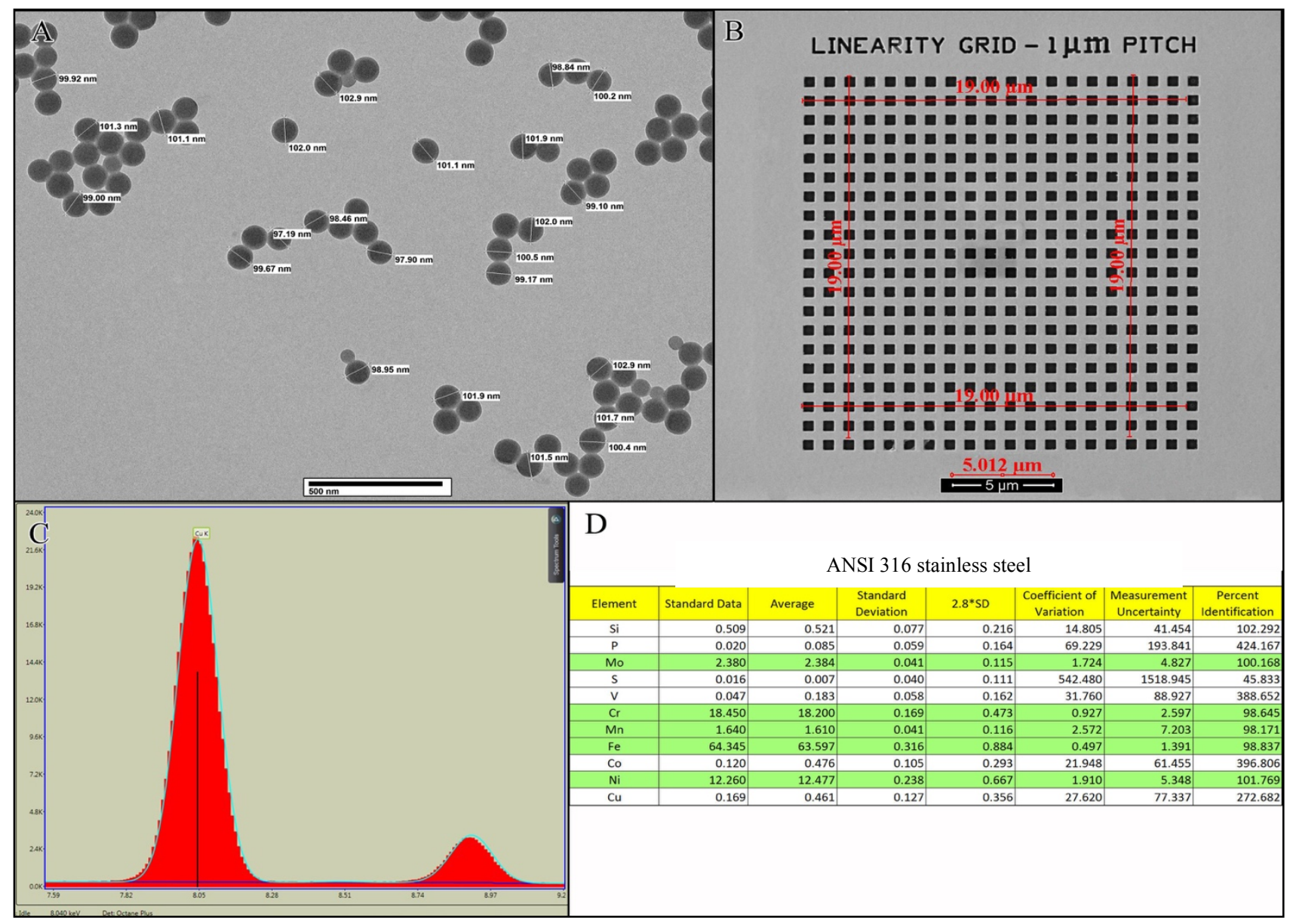

Figure 1. A) TEM standard: NIST traceable Nanospheres; B) SEM standard: Geller MRS-5; C) EDS spectrum showing Copper K line at $8.04 \mathrm{eV}$; D) EDS statistical data from SRM 1155. 